\title{
LA ARQUEOLOGÍA EN LA ENSEÑANZA OBLIGATORIA. EL EJEMPLO DEL CAMPO DE APRENDIZAJE DE LA NOGUERA
}

\author{
Antoni Bardavio Novi y Sònia Mañé Orozco ${ }^{2}$
}

\section{Resumen:}

El Campo de Aprendizaje de la Noguera es un servicio educativo del departamento de enseñanza de la Generalitat de Catalunya que tiene como ejes básicos de su actividad didáctica la arqueología y la prehistoria. Recibe alumnado desde educación infantil (3 años) a bachillerato (17 años), en periodo escolar. Destaca entre sus propuestas curriculares el aprendizaje a partir de la excavación de yacimientos arqueológicos simulados. Para desarrollar esta propuesta educativa se proponen cuatro niveles distintos de acercamiento que ajustan el nivel de lenguaje y los objetivos a conseguir al alumnado de los diferentes ciclos educativos participantes. Las actividades relacionadas con la arqueología prehistórica se desarrollan en el Parque Arqueológico Didáctico de Sant Llorenç de Montgai (Lleida), centro de divulgación escolar y de investigación en relación a la hemos acordado en denominar experimentación didáctica en arqueología.

\section{Palabras clave:}

Arqueología, prehistoria, educación, didáctica, ciudadanía.

\section{Abstract:}

The Noguera Learning Camp is an Educational Service provided by the Department of Education of the Government of Catalonia which bases its didactic activity on archaeology and prehistory. It welcomes students ranging from primary school (3 years) up to secondary education (17 years), throughout the academic year. From among its curricular proposals we highlight learning from excavations carried out at simulated archaeological sites. For developing this educational proposal, four different levels of approach have been proposed, these have adjusted the language level and objectives to the students within the different participating educational cycles. The activities relating to prehistoric archaeology are carried out at the Sant Llorenç de Montgai Didactic Archaeological Park (Lérida), an information centre for scholars and research in relation to which we have agreed to refer to as didactic experimentation in archaeology.

\section{Key words:}

Archaeology, prehistory, education, didactic, citizenship.

1 Campo de Aprendizaje de la Noguera. Departamento de Enseñanza de la Generalitat de Cataluña / Profesor en la Unidad de Didáctica de las Ciencias Sociales de la UAB. abardavi@xtec.cat

2 Profesora del Departamento de Enseñanza de la Generalitat de Cataluña / Asociación Cardium Educarq smane@xtec.cat 


\section{El papel de la arqueología en la enseñanza no universitaria}

El desarrollo disciplinar de la arqueología, "la arqueología que se piensa", ha tenido su reflejo en el papel de la arqueología en el marco escolar propiciando diferentes modelos didácticos con su inclusión en la enseñanza-aprendizaje de la historia. La "arqueología que se enseña" responde pues a esta evolución epistemológica. Investigación y escuela también, en el caso de la arqueología, han mantenido y mantienen una estrecha relación, convirtiéndose la divulgación escolar en el altavoz prioritario de aquellas finalidades que desde la investigación académica se ha otorgado al trabajo arqueológico.

Hoy en día, los procedimientos, las habilidades históricas, son el eje básico a desarrollar con la inclusión de la arqueología en los currícula educativos. El carácter manipulativo y experimental y, al mismo tiempo, intelectual de la actividad arqueológica convierten su inclusión en la enseñanza obligatoria en un instrumento importante para desarrollar una enseñanza de la historia que permita al alumnado ser partícipe de un aprendizaje no estático y meramente receptivo, sino participativo, que potencie el aprendizaje significativo, que los acerque al hecho histórico rompiendo las barreras existentes con el libro de texto y la simple recepción de conocimientos cerrados e inamovibles.

Más allá de la identificación y clasificación de fuentes primarias, que se derivan de la realización de actividades encaminadas a trabajar en el aula los contenidos procedimentales, el énfasis en los procedimientos estimula al alumnado a ver a los arqueólogos como detectives del tiempo. La disciplina arqueológica llena de contenidos procedimentales, generados por la propia metodología del trabajo arqueológico, la enseñanza de la historia en las aulas no universitarias, hasta el punto de convertir los procedimientos en eje del proceso cognitivo del alumnado.

La arqueología también forma parte integral del medio. Por lo tanto, como representación viva, en cuanto a pasado que tenemos presente en nuestro entorno cercano, propicia la entrada de aprendizajes sociales en el aula. La arqueología puede convertirse en un instrumento que estimule el desarrollo de capacidades de formulación de hipótesis de trabajo, de búsqueda de fuentes históricas, de desarrollo de capacidades críticas del alumnado.

Por último, la arqueología tiene relación, especialmente, con la comprensión de la actividad humana a lo largo del tiempo, por lo tanto se refiere a la explicación de la actividad humana. Requiere una comprensión de la amplitud del esfuerzo humano, con una atención preferente a los restos materiales de este esfuerzo que han sobrevivido a actividades posteriores en el decurso del tiempo.

De forma esquemática podríamos decir que con la inclusión de la disciplina arqueológica en el ámbito escolar se pretende: 
- Recurrir a un modo de presentar la información más próxima y comprensible al alumnado, proponiendo una historia más viva y menos inmutable.

- Implicar de forma activa al alumnado en el trabajo de investigación y en la construcción del conocimiento histórico. Conseguir una concepción elaborada de la realidad histórica. No se trata de que los alumnos hagan de arqueólogos sino de que aprendan historia.

- Fomentar en el alumnado la adquisición de un pensamiento social crítico, es decir, aquel tipo de pensamiento que comporta el dominio de habilidades cognitivas del tipo formulación de hipótesis, sintetizar, predecir o valorar ideas y situaciones. Lo que podemos definir como antídoto contra la propaganda, los estereotipos y los prejuicios.

Por supuesto, la edad de los alumnos condiciona la realización de determinadas actividades. Hace falta, pues, señalar diferentes niveles de acercamiento a las evidencias arqueológicas en función de las edades con las que enseñamos y aprendemos. Ante un resto material del pasado podemos establecer cuatro niveles de aproximación al pasado/presente:

1. Reconocer un resto del pasado.

2. Entenderlo y por lo tanto ser capaz de ordenarlo en una sucesión simple de más antiguo a más moderno.

3. Interpretarlo en función de sus características morfológicas y funcionales.

4. Relacionarlo con una sociedad y situación histórica determinada, sabiendo pasar del plano político y económico global, al mundo de lo local y cotidiano, y al revés.

La arqueología permite desarrollar un currículum transdisciplinar que, entre otros, puede basarse en la resolución de problemas específicos mediante el desarrollo de proyectos de investigación utilizando fuentes diversas que se pueden realizar en pequeños grupos que trabajen por proyectos. En este modelo es el alumnado quien toma el protagonismo del proceso de aprendizaje en el cual el papel desempeñado por el profesorado se desarrolla como guía y recurso.

\section{Arqueología, experimentación y metodologías didácticas}

El método en didáctica corresponde al procedimiento o conjunto de procedimientos que organizan los elementos del proceso de enseñanza/ aprendizaje para conseguir que el alumnado interiorice los nuevos conocimientos 
de forma eficiente y duradera. Numerosos pedagogos, psicólogos y sociólogos de la educación han profundizado desde el Renacimiento en propuestas sobre los procesos cognitivos en las aulas:

- Comenius (1592-1670), ya hablaba de tres momentos en el proceso de instrucción: autopsia, la visión, la observación y el análisis de aquello que debe aprenderse; autocracia, la fase de retención y asimilación interior (relacionar) de cuanto se ha observado por los sentidos o las explicaciones; por último, la autopraxia, o interiorización, asimilación y comunicación (aplicación), de cuanto se ha aprendido.

- Pestalozzi (1746-1827), uno de los pioneros de la escuela activa, intentó demostrar que el método más adecuado para enseñar es aquel que tiene como punto de partida la espontaneidad y la intuición, mediante la observación y la imitación de aquello que rodea a los alumnos en su entorno cotidiano.

- Decroly (1871-1932) proponía un modelo de procedimiento didáctico en tres fases para desplegar cada centro de interés: observación, asociación y expresión de lo que se ha aprendido de forma elaborada, estructurada e integrada en su persona.

Podemos referirnos al relieve que debe tomar en la enseñanza de la historia la formación metodológica, como vía para dotar al alumnado de unas determinadas capacidades de análisis racional con una utilidad genérica para su desarrollo como individuos. En este sentido, remarcamos el papel que pueden jugar las propuestas de simulación, entendiéndolas como la recreación o reconstrucción de un determinado proceso o de una determinada casuística histórica. Los elementos más relevantes para un método basado en estrategias de simulación son:

- Partir de la realidad más cercana.

- Simular la realidad cuando ésta no es accesible.

- Desarrollar la observación y el análisis.

- Entender la reflexión como motor de cambio.

- Ampliar significados e intereses.

- Relacionar teoría y práctica.

- Favorecer la transferencia.

El acercamiento escolar a la comprensión de las fuentes materiales desde la simulación implica la introducción de experimentaciones arqueológicas en las aulas. 
Desde el ámbito estricto de la investigación académica, la experimentación puede considerarse como un elemento fundamental y crítico de la arqueología como investigación del pasado. Se ha utilizado como instrumento mediante el cual plantear preguntas a los restos del pasado. La respuesta a estas preguntas estimula nuevamente el proceso que se considera como causa; estructura una hipótesis. El experimento se configura como la constatación de que una interpretación tiene base real en el hecho concreto. Una vez realizada la experiencia hace falta comparar los resultados obtenidos con los datos arqueológicos. Si no existe correlación entre estos resultados y las hipótesis o interpretaciones, éstas tienen que desecharse, no sólo como no válidas sino como positivamente erróneas. De una hipótesis o interpretación errónea surgen hipótesis o interpretaciones alternativas.

De hecho, mediante la arqueología, el aprendizaje de la historia cuenta, a diferencia de los métodos de la historiografía, con un potencial laboratorio en el cual reproducir y manipular elementos tangibles que permitan hacer una aproximación más fidedigna a los fenómenos sociales. El proceso experimental tiene una metodología estricta. La interpretación o hipótesis basada en los datos arqueológicos está sujeta a pruebas empíricas, destinadas a probar o negar la hipótesis y que utiliza las normas estrictas de la ciencia implicada, tanto en la construcción de la prueba como en el análisis de los resultados. Sin embargo, otra peculiaridad de la arqueología consiste en que es una de las pocas disciplinas que en el proceso de obtención de sus datos, al mismo tiempo, se destruye la evidencia. Únicamente realizando una prueba sobre la interpretación de los restos puede desarrollarse algún tipo de verificación. El experimento se convierte, por lo tanto, en un proceso de reajuste de la interpretación.

\section{Relevancia educativa de la prehistoria}

Denominamos relevancia educativa a aquellos aprendizajes que desde la prehistoria pueden aportar elementos formativos a los niños y jóvenes de cualquier etapa de la educación obligatoria, que tiendan a formarlos como futuros ciudadanos y ciudadanas. En concreto, rehuir de la arqueología prehistórica como finalidad educativa en ella misma, intentando buscar contenidos que permitan al alumnado entender la sociedad actual en la que viven y participar en ella de forma activa.

Tenemos que buscar en la investigación prehistórica argumentos educativamente relevantes, que se formulen no en términos disciplinares sino en relación a cuestiones interdisciplinares de significación social. Frecuentemente, la razón de ser, casi única, de la prehistoria se define en términos de la antropología física como la búsqueda de la definición de la naturaleza humana o como procedimiento de construcción/deconstrucción de las narrativas sobre los 
orígenes (de las desigualdades sociales, del estado, de la religión, del arte,...). En cambio, una reflexión sobre la investigación prehistórica puede mostrar que muchos de los aspectos que tratamos desde una perspectiva disciplinar pueden formar parte de discursos argumentales sobre cuestiones relevantes desde el punto de vista educativo.

Es en este sentido que ayuda a comprender muchos de los elementos que configuran nuestra sociedad actual. El elemento primero y principal; ¿qué nos define como seres humanos? Lo humano se define, según esta perspectiva educativa, por dos grandes principios: la capacidad para diseñar y crear las herramientas que le faciliten la subsistencia (el principio tecnológico); y por ser un ser eminentemente social, con una necesidad vital de relacionar-se permanentemente con otros humanos (familiares, amigos y amigas, compañeros y compañeras,...) para su supervivencia. Esto será lo que promoverá en el ser humano desde tiempos prehistóricos, el desarrollo de sistemas complejos de comunicación (gestuales, orales, musicales, icónicos y, más tarde, escritos), así como la aparición del pensamiento simbólico y abstracto.

También fue en la prehistoria cuando se desarrollaron procesos importantes para entender nuestro mundo actual, tanto en lo referente a la relación entre las personas y los pueblos, como en muchas de las formas de la economía actual. Por ejemplo, los profundos cambios que sufrieron personas y sociedades con el paso del paleolítico al neolítico, permiten al alumnado actual profundizar en conceptos temporales esenciales en la construcción de la concepción del tiempo como son; la sucesión, la posición en el tiempo, la reversibilidad, la simultaneidad, la continuidad y el cambio.

Así mismo, la propia naturaleza de la investigación arqueológica en prehistoria, permite el fomento de habilidades vinculadas a la indagación: identificar; observar, preguntar, localizar, obtener, analizar y representar información; plantear y contrastar hipótesis y realizar inferencias. Estos contenidos de tipo procedimental pueden ser desarrollados a partir del uso didáctico de fuentes materiales como herramientas para reconocer el pasado desde la que hemos denominado experimentación didáctica en arqueología como una aproximación al pasado prehistórico, a partir de la reconstrucción de procesos tecnológicos y lenguajes del pasado.

\section{¿Qué es la experimentación didáctica en arqueología?}

La investigación arqueológica basada en la experimentación se fundamenta en la simulación, es decir, la realización de comprobaciones o series de observaciones a partir de la intervención sobre materiales que simulan situaciones o procesos históricos. De forma análoga y desde el punto de vista de la metodología educativa, las características de las propuestas didácticas fundamentadas en la arqueología experimental coinciden con las de la práctica del método de simulación que 
consisten en la observación, el análisis y la reflexión sobre los restos materiales del pasado y su interpretación para reproducirlo, su representación física, la subsiguiente acción sobre la representación con una gran participación interdisciplinar y la valoración de los efectos que esta acción produce sobre los aprendizajes humanos. A pesar que ambas se caracterizan por tratarse de simulaciones, las finalidades educativas de la experimentación arqueológica aplicada al ámbito de la educación básica formal en las escuelas e institutos, son transversales ya que lo que se pretende, además de incidir en estrategias de enseñanza/aprendizaje en los campos disciplinares de las ciencias sociales, es desarrollar la capacidad de imaginar y representar, de promover estrategias con las cuales el alumnado pueda enfrentarse a una situación donde se tienen que tomar decisiones, sean arqueológicas o no.

Por lo tanto, la experimentación didáctica en arqueología pretende propiciar:

- La comprensión del proceso de investigación desarrollada por la arqueología, y que permite comprender las sociedades pasadas a partir de la observación, análisis e interpretación de sus restos materiales.

- Un acercamiento del alumnado al pasado prehistórico a partir de la reconstrucción y reproducción de procesos tecnológicos y del mundo simbólico de aquellas sociedades, com una clara voluntad empática.

\section{La experiencia del Campo de Aprendizaje de la Noguera}

Los Campos de Aprendizaje son servicios educativos del Departamento de Enseñanza de la Generalitat de Cataluña que ofrecen la posibilidad de hacer estancias de dos a cinco días, o salidas de trabajo de un día al alumnado de los centros docentes de Cataluña de educación infantil, primaria y secundaria. En estos momentos la red de campos de aprendizaje está compuesto por dieciséis centros que tienen como objetivos prioritarios; promover actividades educativas innovadoras en espacios naturales y culturales emblemáticos del territorio catalán, participar en la formación inicial y permanente del profesorado, y participar en la investigación de aquellos temas clave de los propios campos de aprendizaje. Estos permiten el desarrollo de actividades educativas en entornos reales, prolongando el currículum escolar fuera de las aulas, en laboratorios vivenciales que promueven la aplicación de conocimientos vinculados a las áreas de ciencias naturales y ciencias sociales de manera transversal e interdisciplinaria.

El Campo de Aprendizaje de la Noguera pretende proporcionar al alumnado las herramientas educativas necesarias que les permitan interpretar y comprender porque Sant Llorenç de Montgai -localidad de la comarca leridana de la Noguera donde se encuentra ubicado- ha sido un entorno ocupado por el ser humano 
desde la prehistoria debido a sus excepcionales características orográficas y a su riqueza natural. La explotación de los recursos que la naturaleza ofrece en este paraje constituyó la base de las formas de vida de los cazadores, recolectores y pescadores del paleolítico.

El proyecto educativo del CdA de la Noguera está vinculado al yacimiento paleolítico de la Roca dels Bous (Sant Llorenç de Montgai). Cabe resaltar el trabajo conjunto que desarrollan el profesorado del Campo de Aprendizaje con el grupo de arqueólogos del Centro de Estudios del Patrimonio Arqueológico de la Prehistoria de la $\cup A B$, que excavan los yacimientos paleolíticos de la comarca. A este sitio arqueológico se suma el Parque Arqueológico Didáctico de Sant Llorenç de Montgai, en el que se reproducen un asentamiento al aire libre del paleolítico superior, una cabaña del neolítico medio, y dos zonas de excavación arqueológica. Estas estructuras permiten desarrollar un proyecto integrado de acercamiento al pasado prehistórico por medio de la arqueología y el uso de fuentes materiales (reproducciones de útiles y herramientas) como recurso didáctico.

La metodología didáctica propuesta en las actividades que llevan a cabo permiten al alumnado interpretar las evidencias arqueológicas siguiendo una estrategia didáctica de simulación, descubrimiento guiado y empatía que facilita la comprensión y valoración de todos los elementos que configuran la fisonomía actual de este territorio y permiten entender su pasado más lejano.

También se incorporan al Proyecto Educativo del CdA de la Noguera, como objetivos específicos de aprendizaje, una serie de competencias básicas referidas a la aplicación de nociones, conceptos científicos y técnicos, y de teorías científicas básicas previamente comprendidas. Esto implica la habilidad progresiva para poner en práctica los procesos y actitudes propios del análisis sistémico y de indagación científica: identificar y plantear problemas relevantes; realizar observaciones directas e indirectas; formular preguntas; localizar, obtener, analizar y representar información cualitativa y cuantitativa; plantear y contrastar hipótesis y realizar inferencias de diferente nivel de complejidad. Todo este planteamiento teórico, aplicado a los objetivos señalados en el proyecto educativo del campo de aprendizaje persigue, en definitiva, el desarrollo del espíritu crítico en la observación de la realidad y en su análisis, así como la potenciación de valores y criterios éticos.

Así pues, en el CdA de la Noguera se lleva a término un proyecto educativo basado en la aproximación del alumnado a la disciplina arqueológica y a la prehistoria que pretende integrar las aportaciones de la investigación, tanto metodológicas como históricas, al conocimiento de este territorio. Las actividades propuestas se basan en lo que hemos denominado "experimentación didáctica en arqueología", para diferenciarlo de la arqueología experimental que se desarrolla en el marco de la investigación prehistórica, al no ser equivalente, como ya hemos explicado, estrictamente a los propósitos y dinámicas de esta. 
La interacción del alumnado con los procedimientos y objetos del pasado -la experimentación didáctica en arqueología- que se propicia en el proyecto del CdA de la Noguera, tiene la voluntad de superar las propuestas educativas basadas en la sucesión de talleres de arqueología con la única finalidad del propio aprendizaje arqueológico y de la prehistoria en ellos mismos, para tender a convertirlas en instrumentos de formación integral de niños y jóvenes respecto a su educación integral como futuros ciudadanos y ciudadanas, mediante estrategias didácticas dinámicas y participativas basadas en la interacción dialogada permanente con las fuentes materiales y el planteamiento de problemas que fuerzan al alumnado a aplicar conocimientos previos adquiridos en el ámbito escolar, a menudo fuertemente disciplinares y desconectados entre ellos, de manera integrada. Así pues, es imposible plantear desde esta perspectiva, actividades basadas en la observación pasiva de demostraciones, y en la transmisión de conocimientos sabios ya elaborados en discursos magistrales. El alumnado se convierte de esta manera en constructor o reconstructor del conocimiento aprendido mediante las actividades propuestas.

\section{¿Cuánto tiempo hace?}

Esta actividad se desarrolla en el bloque referente a Experimentación didáctica y metodología histórica, y en concreto en el ámbito Excavar el pasado. De hecho es la actividad previa a la excavación simulada de un yacimiento arqueológico de época prehistórica que debe promover en el alumnado el conocimiento de los procedimientos de trabajo que permiten obtener información del pasado desde el trabajo de campo en arqueología. Ésta debe permitir entender concretamente que principios geológicos se producen en la superficie terrestre para hacer que los restos del pasado se encuentren actualmente enterrados, y que haga falta por lo tanto, excavar para localizarlos y estudiarlos para extraer información histórica.

El recurso didáctico empleado es una estratigrafía simulada de tamaño real, ante la cual se sitúa al alumnado para establecer un diálogo, fomentando la observación y la interacción basada en dar respuesta a preguntas/problema. No se trata pues de interpretar una estratigrafía real, sino una creada para aprender.

Esta estratigrafía que reproduce estructuras características de diferentes momentos de la historia de la comarca de la Noguera, permite reconocer el tiempo histórico a partir de principios estratigráficos básicos: como se produce la acumulación sucesivas de los sedimentos que forman los estratos; cuál es en principio el estrato más antiguo, el que se encuentra en una posición superior o en una superior; qué nos señala la datación relativa de una estructura en un yacimiento arqueológico; qué métodos existen para datar restos arqueológicos de forma absoluta;... 


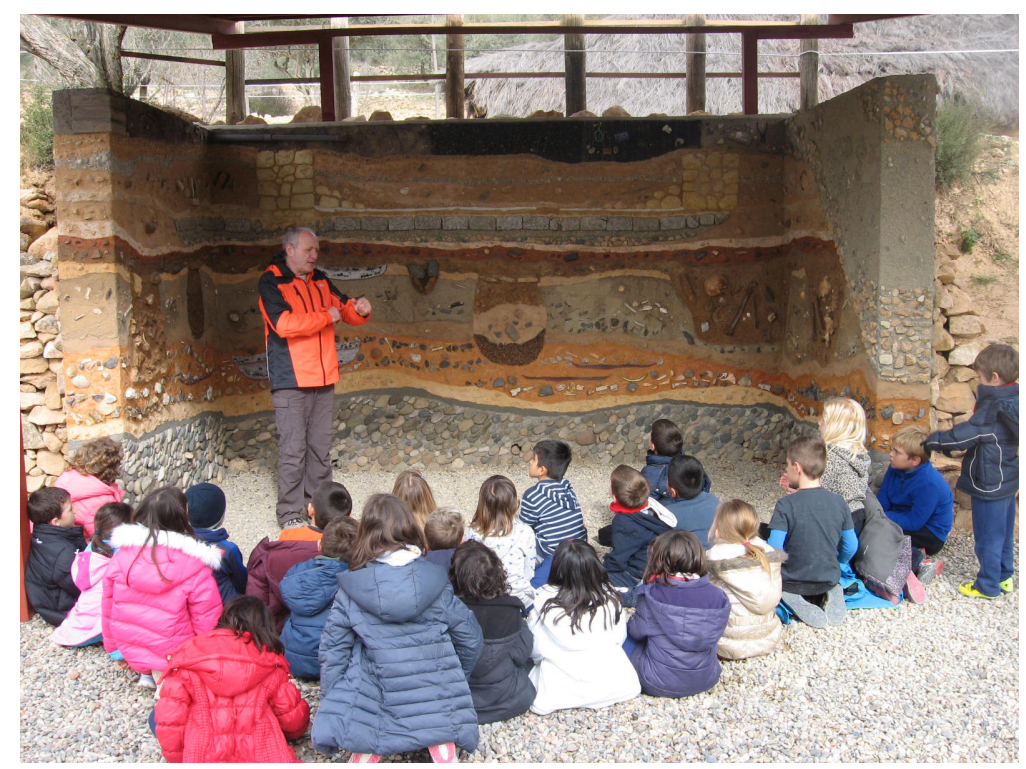

Evidentemente en esta actividad se hace un tratamiento adaptado a los diferentes grupos de edades desde primero de educación primaria hasta bachillerato. No en todos los niveles educativos se trata todo, ni de la misma manera. Tanto el nivel de lenguaje utilizado, como las observaciones y preguntas que se plantean, están en relación a la edad del alumnado y al currículum educativo propuesto para el nivel escolar en el cual desarrollan su actividad escolar de aprendizaje.

Un acercamiento simple, dirigido a alumnado de 6 a 8 años (ciclo inicial de educación primaria), puede partir de la pregunta siguiente: Si los arqueólogos tienen que excavar para encontrar los restos de nuestros antepasados, iquiere esto decir que vivían bajo tierra?. Un primer objetivo en este nivel educativo sería llegar a entender que con el paso del tiempo van superponiéndose capas de tierra, y que por lo tanto no es que antiguamente la gente viviera bajo tierra, sino que el nivel en el que vivían ha quedado tapado con el paso del tiempo por tierra acumulada. Un segundo objetivo sería entender la utilidad del análisis estratigráfico para los arqueólogos. En este caso un paralelismo con una vivencia habitual de los niños y niñas puede ser definitivamente esclarecedor. A la pregunta ¿qué tenéis que hacer cuando os regalan un pastel el día de vuestro cumpleaños para saber de qué está hecho?, la respuesta es cortarlo por la mitad y observar las diferentes capas (bizcocho, chocolate, mermelada, crema, nata,...). Esto acerca al alumnado a la comprensión de la estratigrafía como una herramienta que permite a los arqueólogos conocer si vivieron personas en ese lugar, y en qué momento de la historia lo hicieron.

Una aproximación con alumnos de 9 a 12 años (ciclos medio y superior de educación primaria y primer curso de educación secundaria obligatoria) nos 
permitiría ya poner en juego la aplicación de conocimientos curriculares en la respuesta de la misma pregunta. La dinámica de la superficie terrestre, con la erosión, transporte y sedimentación de materiales, hace que el alumnado de sentido útil a un conocimiento aprendido en un contexto diferente al que se aprende en este momento. Evidentemente la capacidad expositiva y el rigor en la expresión conceptual será diferente entre un niño o niña de 9 años y otro u otra de 12.

\section{Excavando el pasado}

Este conjunto de actividades tienen como elemento esencial la excavación de un yacimiento arqueológico simulado. Los objetivos didácticos se centran en el conocimiento del método arqueológico del trabajo de campo, la práctica del registro arqueológico de los materiales localizados y la interpretación del yacimiento a partir del análisis de los objetos encontrados por el alumnado y su distribución espacial en el yacimiento simulado. Las actividades que se realizan refuerzan la idea del trabajo arqueológico como trabajo cooperativo, como puzzle que requiere de un primer momento de interpretación inductiva de las fuentes materiales encontradas en cada uno de los cuadros en que se divide el yacimiento, y su posterior propuesta interpretativa basada en la formulación de hipótesis.

Se han diseñado materiales didácticos de registro de observación y análisis de cinco niveles diferentes: educación infantil (con un espacio arqueológico diferenciado y singular del cual después hablaremos), ciclo inicial de educación primaria, ciclo medio de educación primaria, ciclo superior de educación primaria y primer ciclo de educación secundaria obligatoria.

Han existido críticas al valor educativo de una propuesta de estas características, argumentando que no aportan aprendizajes significativos en el alumnado. Incluso desde la investigación educativa se ha insinuado el error de formar en un tipo de actividad que potencia "posibles furtivos del futuro".

En el desarrollo de nuestro proyecto educativo, la excavación simulada de un yacimiento arqueológica genera una comprensión esencial sobre cómo se construye el conocimiento científico que potencia el espíritu crítico a partir del desarrollo de capacidades de observación, análisis y comunicación de resultados. Esto la convierte en un recurso educativo esencial para desarrollar una propuesta educativa de tipo competencial. Así mismo, estamos convencidos que conocer el valor explicativo de los restos arqueológicos asegura, precisamente, un rechazo al furtivismo, generando un compromiso social respecto al patrimonio común. 

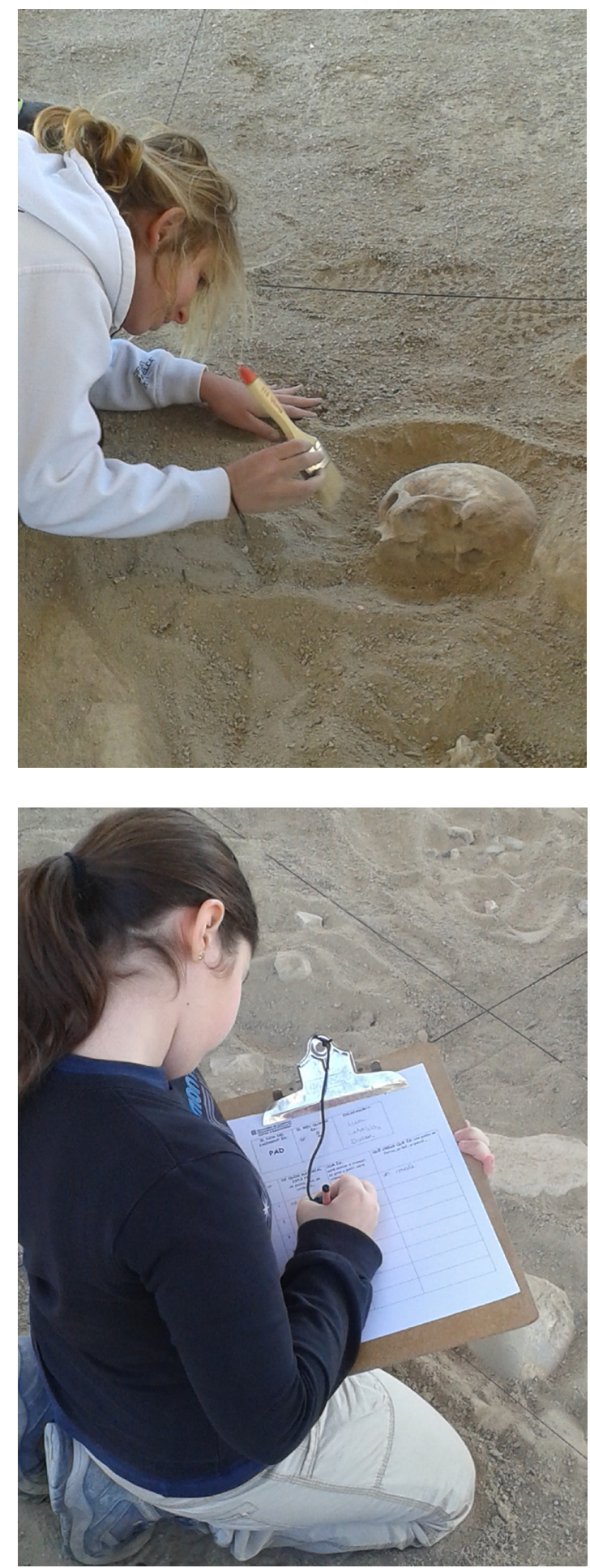

意 


\section{Excavando un yacimiento arqueológico con niños y niñas de 3 a 5 años}

El curso 2013-2014, el equipo docente del CdA de la Noguera se propuso la construcción de un espacio de excavación especialmente pensado para las niñas y los niños del segundo ciclo de educación infantil.

Los objetivos de aprendizaje señalados en esta actividad en ciclos educativos posteriores, tenía absoluta razón de ser con niños y niñas de 3 a 5 años. De hecho, es en esta etapa educativa donde se cimentan y construyen todos los aprendizajes posteriores. Comprender (experimentando) como se construye el conocimiento científico, ser capaces de clasificar los "restos arqueológicos" según la naturaleza de los materiales localizados (herramientas de sílex, de hueso, cantos rodados pintados con óxido de hierro, ocre o carbón,...), registrar los hallazgos en hojas de registro con dibujos y adhesivos y ponerlos en común para entender de forma colectiva, asegura una percepción de la historia constructiva y crítica en esta ciudadanía del futuro.

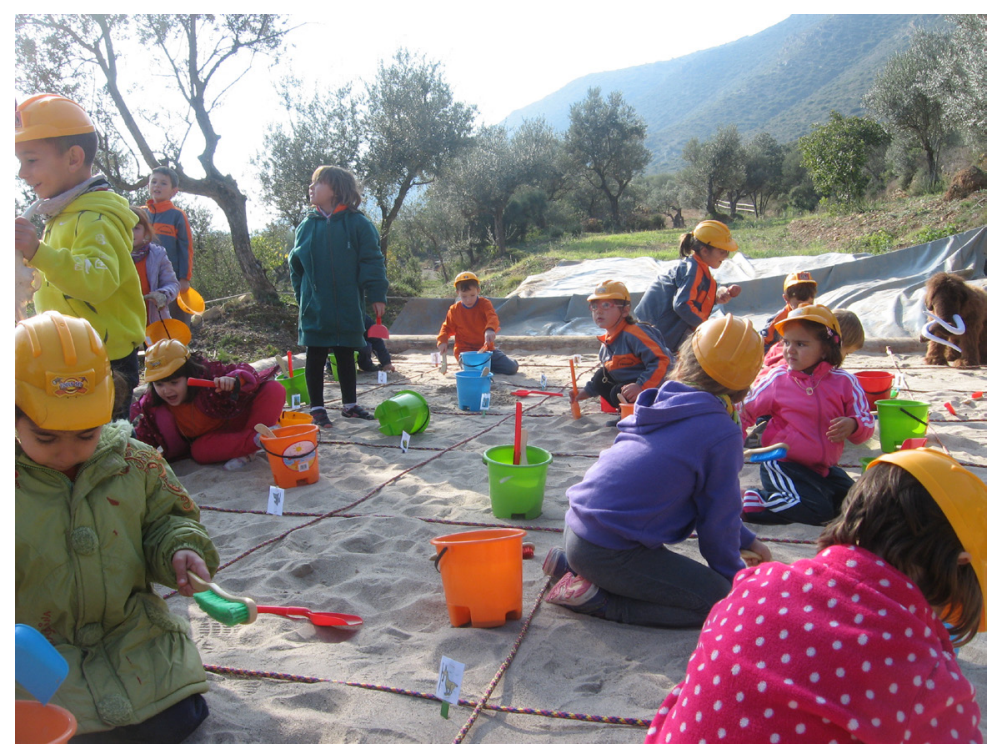

\section{Conclusiones}

Como conclusión fundamental de nuestra experiencia desde el punto de vista didáctico, se puede afirmar que los escolares que participan en propuestas de educación arqueológica de forma activa y experimental en el marco de un entorno natural fuera del aula, desarrollan una serie de actitudes y valores que les permiten modificar o entender conceptos previos (más o menos estereotipados), 
haciéndoles conscientes de aquellas concepciones erróneas que tenían y proporcionándoles elementos de análisis que les permitan cambiarlas o o ampliarlas , así como utilizar herramientas de análisis en otros ámbitos de estudio. Este cambio de actitud hacia el conocimiento social e histórico es difícilmente abarcable en el marco de procesos de enseñanza/aprendizaje de tipo transmisor, en los cuales el alumno es un simple espectador de aquello que se le muestra y que, por lo tanto, suele quedar excluido de un tratamiento afectivo, y por lo tanto ineficaz, de aprehensión de nuevos conocimientos.

Resulta indispensable en un proyecto como el del CdA de la Noguera que se cumplan una serie de requisitos que lo hacen viable desde un punto de vista didáctico:

- Un marco de aprendizaje que genera un ambiente en el cual el alumnado desee actuar y proponer que desarrollen actitudes positivas en la acción, contagiando el deseo de aprender.

- Una metodología didáctica innovadora, basada en el "saber hacer" y en el "saber interpretar y explicar" del alumnado, donde hace falta enseñarlos a organizar y a reconstruir la realidad porque cada uno de ellos y ellas den sentido a su propia experiencia.

- Unos objetivos didácticos marcados de forma especial por la observación y análisis transdisciplinar del entorno, objeto de estudio del alumnado, que los ha de poner en situación para aprenderá construir modos de abordar problemas y darles solución.

- Una organización de las actividades didácticas basadas en el trabajo cooperativo -característica compartida con el propio trabajo arqueológico-, convirtiendo este hecho educativo en una herramienta más de aprendizaje de la vida en grupo.

- Una interacción sistemática entre investigación científica e investigación escolar, que facilite la transposición didáctica de los contenidos científicos.

- Un espacio didáctico adecuado para desarrollar actividades vinculadas a la experimentación histórica y a los procesos de empatía.

En definitiva, la propuesta del CdA de la Noguera pretende desarrollar, a partir del conocimiento sobre la evolución y organización de las sociedades a partir de la información que aporta la investigación arqueológica, la capacidad para realizar juicios éticos, para escoger y tomar decisiones que potencien la participación y el ejercicio activo, responsable y comprometido de los derechos y deberes como ciudadanos y ciudadanas. Favoreciendo esta propuesta la comprensión de la realidad histórica y social, su evolución, sus éxitos y problemas, que comporta recurrir al análisis multicausal y sistémico para juzgar los hechos y problemas sociales e históricos desde la perspectiva de una comprensión crítica de la historia. 


\section{Bibliografia}

J. SANTACANA, X. HERNÁNDEZ: Enseñanza de la arqueología y la prehistoria. Editorial Milenio. Lleida: 1999

H. COOPER: Didáctica de la historia en la educación infantil y primaria. Ministerio de Educación, Cultura y Deporte / Morata Ed. Madrid: 2002.

A. BARDAVIO A., P. GONZÁLEZ: Objetos en el tiempo. Las fuentes materiales en la enseñanza de las ciencias sociales. ICE UB / Editorial Horsori. Barcelona : 2003.

A.BARDAVIO, P. GONZÁLEZ, J. PIZARRO González P. (2013) "Experimentació didàctica en arqueologia. El projecte educatiu del Camp d'Aprenentatge de la Noguera". Experimentación en arqueología. Estudio y difusión del pasado A. PALOMO, R. PIQUÉ, XAVIER TERRADES EDS. Sèrie Monogràfica del MAC. Girona, 2013:25-30.

A. BARDAVIO, S. MAÑÉ:"Tocant la prehistòria" en GUIX 413. Ed. Graó. Barcelona, marzo 2015: 28-32

A.BARDAVIO, S. MAÑÉ: "Excavar un jaciment arqueològic simulat amb nens de tres a cinc anys? Una experiència didàctica vinculada al temps històric al Camp d'Aprenentatge de la Noguera". Guix Infantil 82. Ed. Graó. Barcelona, septiembre- octubre 2015:19-22. 
